\title{
FINISHING OF TUBES USING BONDED MAGNETIC ABRASIVE POWDER IN AN ABRASIVE MEDIUM
}

\author{
Palwinder Singh, Lakhvir Singh, Sehijpal Singh
}

\begin{abstract}
Magnetic abrasive flow finishing (MAFF) is an unconventional process capable of producing fine finishing with machining forces controlled by a magnetic field. This process can be utilized for hard to achieve inner surfaces through the activity of extrusion pressure, combined with abrasion activity of a magnetic abrasive powder (MAP) in a polymeric medium. MAP is the key component in securing systematic removal of material and a decent surface finish in MAFF. The research background disclosed various methods such as sintering, adhesive based, mechanical alloying, plasma based, chemical, etc. for the production of bonded MAP. This investigation proposes bonded MAP produced by mechanical alloying followed by heat treatment. The experiments have been conducted on aluminum tubes to investigate the influence of different parameters like magnetic field density, extrusion pressure and number of working cycles. The bonded magnetic abrasive powder used in MAFF is very effective to finish tubes' inner surfaces and finishing is significantly improved after processing.
\end{abstract}

Keywords: magnetic abrasive flow finishing, abrasion, mechanical alloying, sintering, aluminium tubes

\section{INTRODUCTION}

High precision finished surfaces is the critical requirement of high technology manufacturing industries. Inner surface finishing of tubes is of the utmost necessity in numerous applications, including high purity liquid and gas flow systems $[1,2]$. But, finishing of these surfaces includes staggering expense and controlled environment during finishing. To meet the prerequisites of the industry, new techniques are being developed consistently. Magnetic abrasive flow finishing (MAFF) is one of such technique in which the material is evacuated so that the surface finishing and deburring is accomplished with the nearness of a magnetic field in the machining zone. This process can be utilized for hard to achieve inner surfaces through the activity of extrusion pressure, combined with the abrasion activity of the polymeric laden medium with magnetic abrasive powder (MAP). This is one of the feasible techniques for producing surfaces of nano and micro level finish. This technique came into existence in early 2000's. The various input process parameters related to MAFF include magnetic field density (MFD), extrusion pressure, abrasive mesh size, abrasive material \& composition and number of working cycles. MAP affects the finishing effectiveness and the final quality of surface. The ferromagnetic particles used in all studies of MAFF are iron $(\mathrm{Fe})$; the abrasives are mainly aluminum oxide, $\mathrm{SiC}, \mathrm{B}_{4} \mathrm{C}$, diamond. MAP can be simply mixed or bonded magnetic particles. A past research study demonstrated that heat treated MAP shows a surface finish higher than normally with mixed particles on most materials.

\footnotetext{
Palwinder Singh, Lakhvir Singh: Mech. Engg. Department, BBSB Engg. College, Fatehgarh Sahib, India
} Sehijpal Singh: Mech. Engg. Department, Guru Nanak Dev Engg. College, Ludhiana, India 
MAP can be of various types such as mechanical mixing of abrasive particles and iron (ferromagnetic) particles, unbonded MAP, sintered MAP, plasma based MAP. The mechanical mixture of abrasive particles and Fe particles include abrasive coated MAP. This technique gives a wide diversity of MAP, but the abrasive layer may get worn off from the magnetic part during the process, subsequently influencing the finishing performance $[3,4]$. The unbonded MAP is a mixture of abrasive and Fe particles. The abrasive is not physically bonded to a Fe particle. In the magnetic field, the magnetic abrasives can move around freely within the constraints [5]. Heat treated MAP have been utilized by the majority of the authors because of its phenomenal surface finishing effects. The particles produced from this technique are moulded at high pressure and high temperature inside an inert gas environment. The subsequent compacts are then crushed and sieved into a particular particle size $[6,7]$. The crushing of compacts brought about the irregular shape of MAP and the improvement of surface finish quality was limited because of the nonuniform depth of cut. To overcome this difficulty, the spherical iron-based magnetic abrasive carrying $\mathrm{Al}_{2} \mathrm{O}_{3}$ particles on the surface were produced by plasma spray method. These magnetic abrasives successfully machined the workpiece. But during machining, the problem of detaching of $\mathrm{Al}_{2} \mathrm{O}_{3}$ particles from the MAP was noticed due to delicate bonding strength between carried $\mathrm{Al}_{2} \mathrm{O}_{3}$ abrasive and $\mathrm{Fe}$ particles in the composite powder [8].

Jain and Adsul [9] machined brass and aluminum as workpieces with abrasive laden medium containing unbonded silicon carbide $(\mathrm{SiC})$ abrasive particles. Jha and Jain [10] prepared abrasive media containing unbonded abrasives (carbonyl iron powder $+\mathrm{SiC}$ abrasives) and finished stainless steel with the prepared abrasive medium in the magnetic rheological AFM process. They found that with increased magnetic field, the surface finish improved gradually. Kar et al. [11] developed SiC abrasive-based media for AFM and found that butyl rubber-based media showed better performance than natural rubber-based media. Kenda et al. [12] used media containing unbonded SiC abrasives for AFM of hardened tool steel AISI D2. They concluded that AFM removes EDM damaged surface and improves the surface finish. Singh and Shan [13] used Brown Super Emery as MAP in the medium to investigate the performance of the MAFF process for finishing of commercially available brass. Singh et al. [14] performed the MAFF process on aluminum and brass as workpieces with abrasive medium containing unbonded $\mathrm{Al}_{2} \mathrm{O}_{3}$ abrasive particles. They concluded that the surface finish was higher for brass, while aluminum does not show any appreciable improvement in surface finish. It was also concluded that the MRR of brass both in AFM and MAFF is higher than aluminum. Sankar et al. [15] used unbonded $\mathrm{SiC}$ abrasive particles in an abrasive medium for AFM of $\mathrm{Al}$ alloy based metal matrix composites (MMCs). They reported that Al alloy/SIC (10\%) MMC showed better $\mathrm{Ra}$ among three workpiece materials by AFM. Tzeng et al. [16] investigated the finishing effect of unbonded $\mathrm{SiC}$ abrasive grains in media for the AFM on micro slit fabricated by wire-EDM. It was reported that surface finish reduced with higher extrusion pressure and decreased with increasing machining time.

The literature shows that most of the researchers had used unbonded abrasive particles in abrasive laden media for MAFF process. No study is available regarding production of mechanically alloyed cum heat treated bonded MAP. So bonded magnetic abrasive particles in abrasive laden media needed to be investigated. This study aims at evaluating the influence of different parameters on the improvement in surface finish of internal surface of small diameter tubes after MAFF. 


\section{PRODUCTION OF BONDED MAGNETIC ABRASIVE POWDER (MAP)}

\section{Mechanical Alloying of Magnetic Abrasive Powder}

MAP is prepared by mixing diamond powder (abrasive particle, mesh size 270) in $30 \%$ by volume in iron powder (ferromagnetic particles, mesh size 300) followed by mechanical alloying (MA) [18]. This is a powder processing method including repeated welding, cracking and rewelding of powder particles in a ball mill/attritor for 6 hours [17]. The photographic view and schematic view of attritor ball mill is shown in Fig. 2(a) and Fig. 2(b) respectively. After MA, very small MAP is acquired in which the abrasive particles are connected to the base metal grid with no holding material, as shown in Fig. 1. Tab. 1 gives the parameters of mechanical alloying.

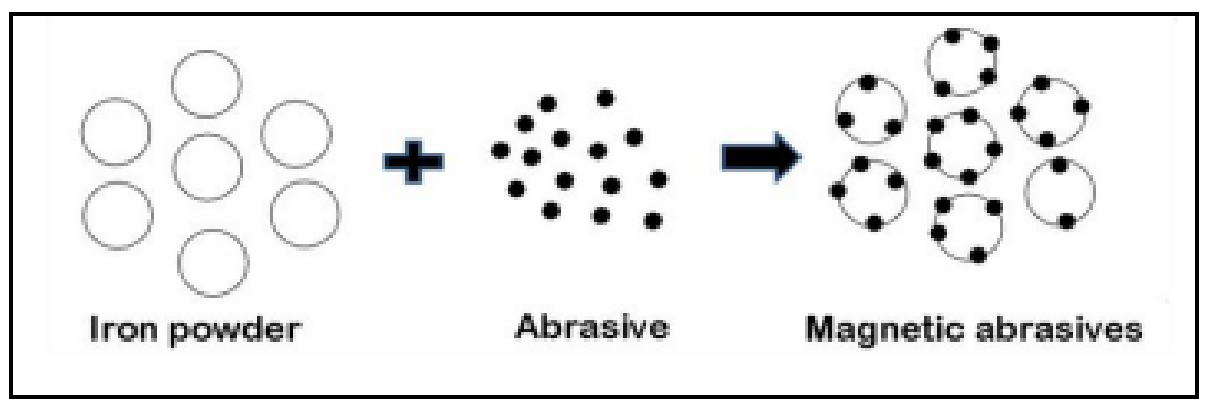

Fig. 1. Schematic view of production of MAP by mechanical alloying method

Tab. 1 MA parameters for MAP preparation

\begin{tabular}{ll}
\hline Parameter & Description \\
\hline Type of mill & Attritor \\
Milling temperature & Room temperature \\
Milling medium & Stainless steel balls of $5 \mathrm{~mm}$ diameter \\
Speed of attritor & $200 \mathrm{rpm}$ \\
Ball to powder ratio & $5: 1$ \\
MA time & 6 hours \\
\hline
\end{tabular}




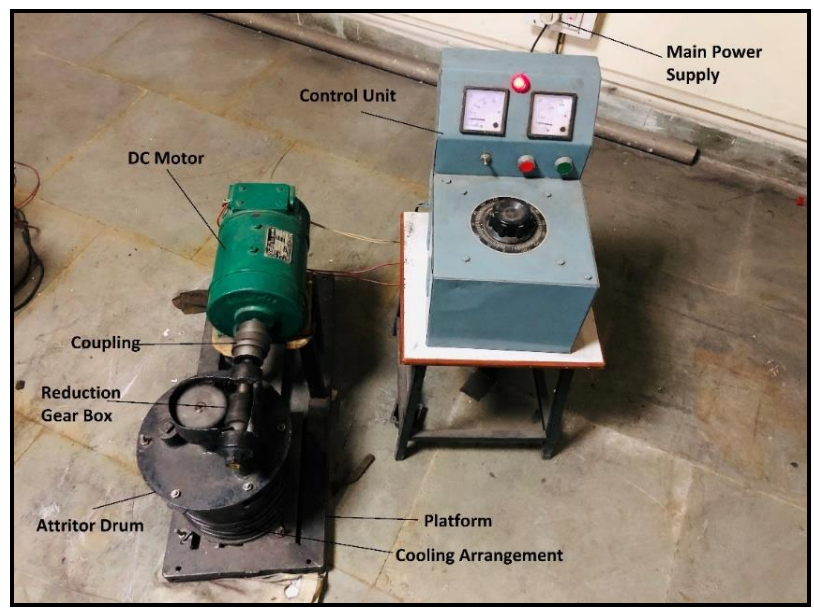

a)

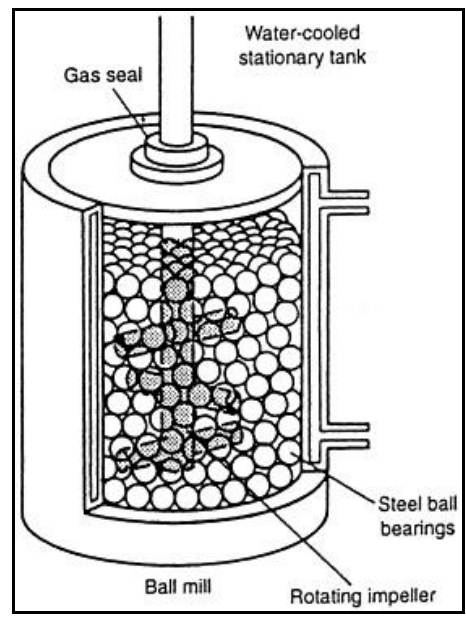

b)

Fig. 2. a) Photographic view of attritor set up and b) schematic view of attritor ball mill.

To identify strength of bonding of magnetic abrasive particles, MAP prepared by MA were subjected to the magnetic separation test. About $5 \mathrm{gm}$ of prepared MAP were shaken out on a sheet of paper and a permanent magnet was passed near the MAP. A complete bonding was noticed, as MAP disappeared from the sheet of paper.

\section{Annealing of Mechanically Alloyed Magnetic Abrasive Powder and Sieving}

Annealing is a heat treatment process in which material is bonded, causing changes in its properties like hardness by heating to a predetermined temperature, holding and finally cooling. The MAP obtained by mechanical alloying is then compressed into cylindrical compacts using an universal testing machine (UTM) in a cylindrical die and annealed in a furnace at $950^{\circ} \mathrm{C}$ in the presence of hydrogen gas (inert environment) for two hours. This results in induced ductility, relieves internal stresses, refines the structure and increases the bonding strength of diamond $+\mathrm{Fe}$ abrasive powder. The magnetic properties of MAP were affected by the annealing temperature. The highest MFD was found to be at $950^{\circ} \mathrm{C}$ [19]. After annealing, the magnetic abrasives were crushed and separated to different sizes using a sieving machine. The magnetic abrasive powder of mesh size 275 was used.

\section{EXPERIMENTAL SETUP AND TEST CONDITIONS}

\section{Magnetic Polymeric Abrasive Medium and Experimental Setup}

The abrasive medium consists of 50\%vol MAP (275 mesh size), 50\%vol base medium (50\%wt polymer and 50\%wt gel). The base medium combination and their percentages were obtained through study of fluid characteristics, their availability and price and some experimental trial and error. A 1:1 proportion of polymer and gel was chosen. Fig. 3 depicts the mechanism of the MAFF process and Tab. 2 depicts the experimental conditions. The internal surface of the aluminum tube was cleaned thoroughly with acetone before and after finishing. The finishing characteristics of produced bonded MAP were analysed by measuring surface roughness $(\mathrm{Ra})$. It was recorded using a Mitutoyo surface roughness tester. The initial value for each piece was $0.90-1.3 \mu \mathrm{m}$. The value of Ra was recorded at four points along the length of tube and percentage improvement in surface 
finish (PISF) was calculated. PISF is defined as the ratio of $\left(\Delta R_{a} \times 100\right)$ and the initial $R_{a}$ value where $\Delta R_{a}$ is the difference between $R$ a values before and after MAFF.

Tab. 2. Magnetic abrasive flow finishing (MAFF) experimental conditions

\begin{tabular}{|c|c|}
\hline Parameter & Description \\
\hline Workpiece & $\begin{array}{l}\text { Commercially available aluminum tube }(\varnothing 16 \mathrm{~mm} \times \varnothing 8 \mathrm{~mm} \times \\
25 \mathrm{~mm})\end{array}$ \\
\hline Magnetic abrasive powder & $\begin{array}{l}\text { Diamond based mechanically alloyed cum heat treated magnetic } \\
\text { abrasive powder ( } 30 \% \text { diamond and } 70 \% \mathrm{Fe} \text { by volume) }\end{array}$ \\
\hline \multicolumn{2}{|l|}{ Constant Parameters } \\
\hline $\begin{array}{l}\text { Gap between pole faces of } \\
\text { electromagnet }\end{array}$ & $30 \mathrm{~mm}$ \\
\hline \multicolumn{2}{|l|}{ Variable Parameters } \\
\hline Magnetic field density & 0.15 to $0.75 \mathrm{~T}$ \\
\hline Extrusion pressure & 1.4 to $7.0 \mathrm{MPa}$ \\
\hline Number of working cycles & $10-30$ \\
\hline
\end{tabular}

Fig. 4 shows the schematic view of experimental set up of magnetic abrasive flow finishing. The hydraulically actuated cylinders extrude magnetic abrasive medium from lower cylinder to finishing space where electromagnets applying an external magnetic field. After a particular distance, limit switch is pressed and hydraulic cylinder stops electrically. After completing the upward stroke, the DC valve operates in the other direction and the pistons start coming downward, pushing the media in the downward direction against the restrictions in the geometry of the fixtures, to obtain the required pressure for abrasion to get the finer finishing in the workpiece. The extrusion pressure was varied from 1.4 to 7.0 $\mathrm{MPa}$. Experiments were conducted on aluminum at magnetic field densities 0.15T, 0.30T, $0.45 \mathrm{~T}, 0.6 \mathrm{~T}$ and $0.75 \mathrm{~T}$, extrusion pressure $1.4 \mathrm{MPa}, 2.8 \mathrm{MPa}, 4.2 \mathrm{MPa}, 5.6 \mathrm{MPa}$ and 7.0 $\mathrm{MPa}$, and 10, 15, 20, 25 and 30 the number of working cycles. 


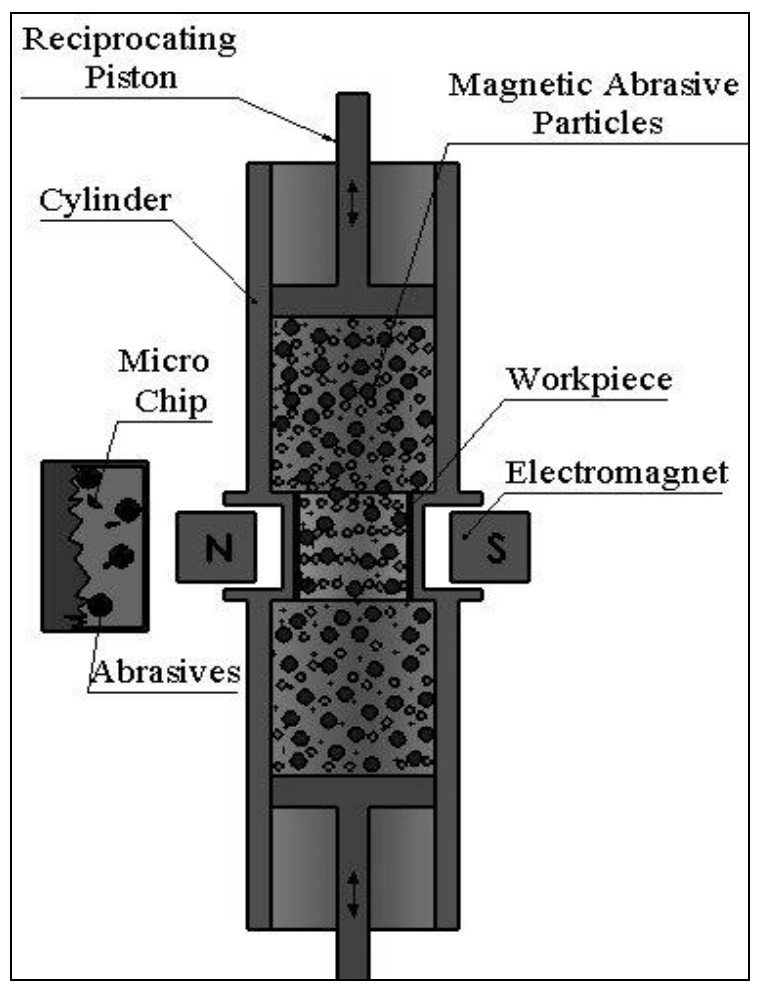

Fig. 3. Mechanism of magnetic abrasive flow finishing proces

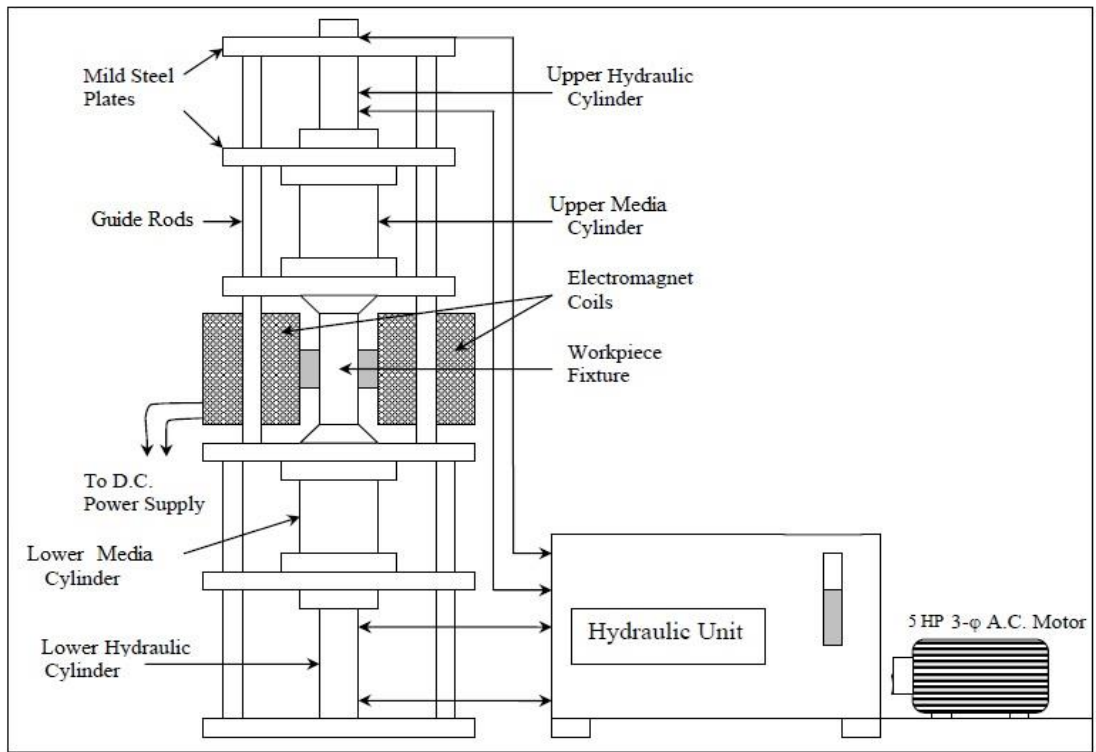

Fig. 4. Schematic view of Magnetic abrasive flow finishing (MAFF) set up. 


\section{RESULTS AND DISCUSSION}

The effects of process parameters: magnetic flux density (MFD), extrusion pressure and number of working cycles on PISF are tabulated in Tab. 3.

Tab. 3. Effect of process parameters on PISF

\begin{tabular}{|c|c|c|c|c|c|c|}
\hline $\begin{array}{c}\text { Exp. } \\
\text { No. }\end{array}$ & $\begin{array}{c}\text { Magnetic } \\
\text { flux density } \\
\text { (Tesla) }\end{array}$ & $\begin{array}{c}\text { Extrusion } \\
\text { pressure } \\
(\mathbf{M P a})\end{array}$ & $\begin{array}{c}\text { Number of } \\
\text { working } \\
\text { cycles }\end{array}$ & $\begin{array}{c}\text { Initial } \\
\mathbf{R a} \\
(\boldsymbol{\mu m})\end{array}$ & $\begin{array}{c}\text { Final } \\
\mathbf{R a} \\
(\boldsymbol{\mu m})\end{array}$ & $\begin{array}{c}\text { PISF } \\
(\boldsymbol{\%})\end{array}$ \\
\hline 1 & 0.15 & 4.2 & 20 & 0.98 & 0.46 & 53.06 \\
\hline 2 & 0.30 & 4.2 & 20 & 1.02 & 0.43 & 57.84 \\
\hline 3 & 0.45 & 4.2 & 20 & 1.01 & 0.37 & 63.36 \\
\hline 4 & 0.60 & 4.2 & 20 & 0.98 & 0.33 & 66.33 \\
\hline 5 & 0.75 & 4.2 & 20 & 1.01 & 0.35 & 65.35 \\
\hline 6 & 0.45 & 1.4 & 20 & 0.91 & 0.53 & 41.75 \\
\hline 7 & 0.45 & 2.8 & 20 & 0.99 & 0.49 & 50.50 \\
\hline 8 & 0.45 & 4.2 & 20 & 1.02 & 0.36 & 64.70 \\
\hline 9 & 0.45 & 5.6 & 20 & 0.90 & 0.30 & 66.67 \\
\hline 10 & 0.45 & 7.0 & 20 & 0.89 & 0.31 & 65.17 \\
\hline 11 & 0.45 & 4.2 & 10 & 0.99 & 0.45 & 54.54 \\
\hline 12 & 0.45 & 4.2 & 15 & 1.01 & 0.42 & 58.41 \\
\hline 13 & 0.45 & 4.2 & 20 & 1.01 & 0.35 & 65.35 \\
\hline 14 & 0.45 & 4.2 & 25 & 0.86 & 0.27 & 68.60 \\
\hline 15 & 0.45 & 4.2 & 30 & 1.00 & 0.47 & 53.00 \\
\hline
\end{tabular}

Because of the data convergence in different finishing conditions, the effects of different parameters are divided into the following three groups:

\section{Influence of Magnetic Flux Density (MFD)}

This set of conditions was considered randomly amongst all feasible conditions: extrusion pressure of 4.2 $\mathrm{MPa}$ and 20 number of cycles and magnetic field densities of 0.15T, 0.30T, 0.45T, 0.6T and 0.75T. The effect of MFD on PISF is shown in Fig. 5. It is clear that with an increase in MFD from 0.15T to 0.60T, the surface finish improves, but after $0.6 \mathrm{~T}$ of MFD, surface finish starts to decline. A possible reason could be that the medium sticks to the workpiece surface strongly and the high hydraulic pressure passes the medium from the middle of the aluminum tube space due to strong magnetic field beside the workpiece walls. This occurs as MAP is stuck to the walls near to the electromagnetic poles. So, in the center of the workpiece, it seems that abrasive medium passes through a passage and there will be no abrasion on the internal workpiece surface. The abrasive medium does not show fruitful material removal by this mechanism which results in less PISF. 


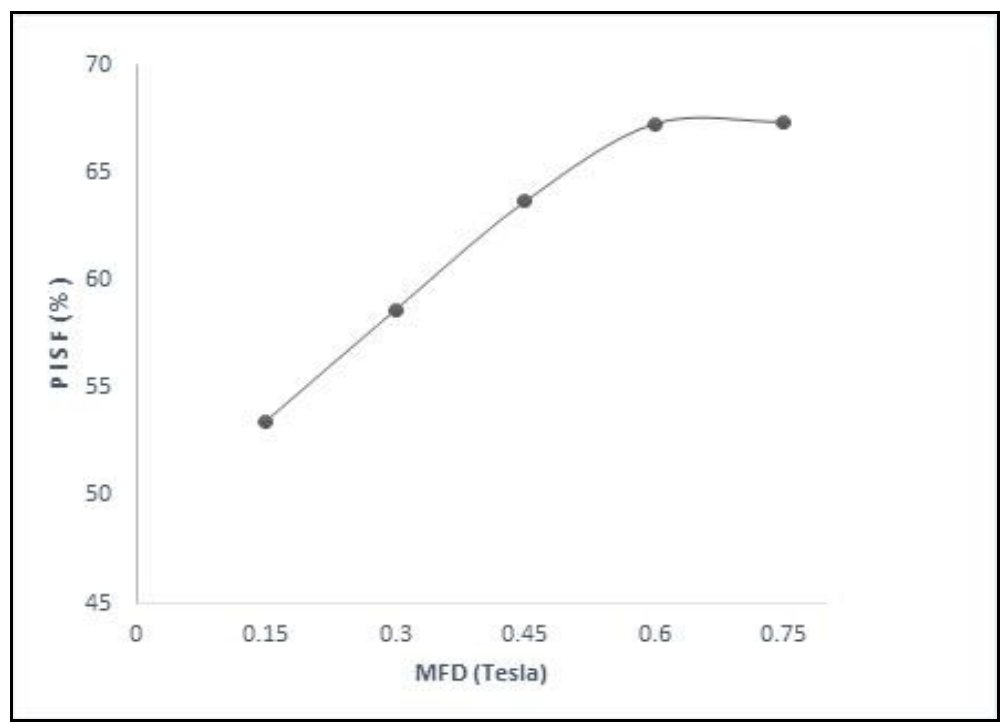

Fig. 5. Influence of magnetic flux density (MFD) on PISF

\section{Influence of Extrusion Pressure}

The process parameters were magnetic field densities of $0.45 \mathrm{~T}, 20$ number of working cycles and extrusion pressure of $1.4 \mathrm{MPa}, 2.8 \mathrm{MPa}, 4.2 \mathrm{MPa}, 5.6 \mathrm{MPa}$ and 7.0 $\mathrm{MPa}$. The results are illustrated in Fig. 6. The improvement in surface finish of aluminum tubes increases while applying extrusion pressure up to 5.6 MPa. As the extrusion pressure increases, it leads to increase in axial force resulting in higher PISF improvements. Further increase in extrusion pressure from 5.6 to $7.0 \mathrm{MPa}$, tends to decrease PISF improvement. This may be due to the fact that at high pressures, number of active abrasive particles becomes less as the highly viscous medium becomes stiffer.

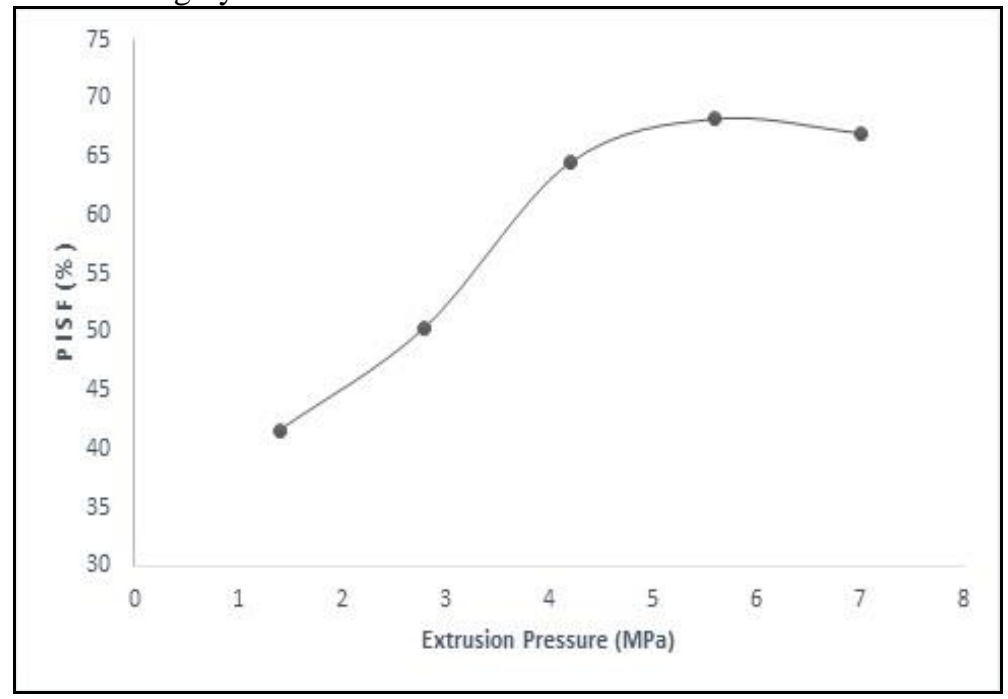

Fig. 6. Influence of extrusion pressure on PISF 


\section{Influence of Number of Working Cycles}

The following conditions were examined: MFD of $0.45 \mathrm{~T}$, extrusion pressure of 4.2 MPa, working cycles 10, 15, 20, 25 and 30. Results are shown in Fig. 7. With increase in working cycles from 10 to 25 , surface finish improves, but after this point up to 30 number of working cycles, surface finish deteriorates. A possible reason could be increased abrasion time which results in removal of more peaks. So, PISF in this region increases and results in a finer surface. Whereas, from 25 to 30 working cycles, extra abrasion time makes finer finished surface, scratched by continuing abrasion after the removal of the majority of the peaks. Fig. 7 represents that during the initial working cycles, PISF increases significantly, further decrease in PISF can be credited to the fact that the sharp abrasive edges get blunt as machining advances.

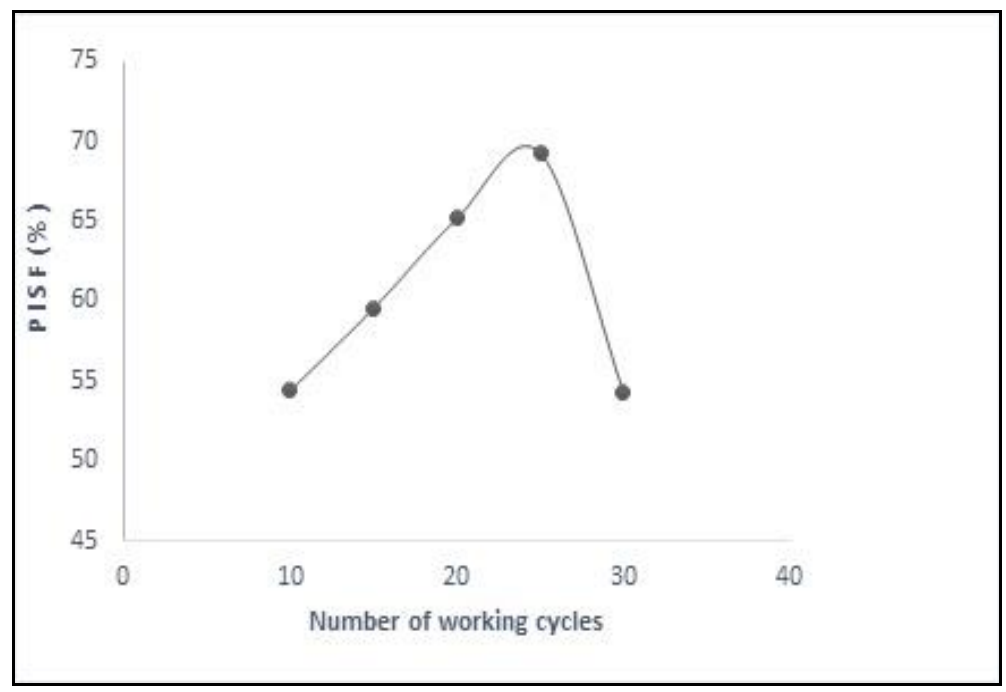

Fig. 7. Influence of number of working cycles on PISF

\section{Scanning Electron Microscopy (SEM) Analysis}

The tube was cut open in the axial direction to evaluate the surface finish by SEM. The scratches produced during the boring process are clearly visible. The boring grooves may not have a systematic pattern in SEM images, but abrasive grain marks made by MAFF are almost parallel. Fig. 8 \& Fig. 9 show surface structure before and after MAFF, respectively. In Fig. 8, it is evident that the surface produced by MAFF is almost fine where porosity does not exist. It is only capable to abrade the edge and create fillet edges of the porosities. In fact, this could not be a deficiency in the parent metal, since MAFF has to remove peaks on the surface. 


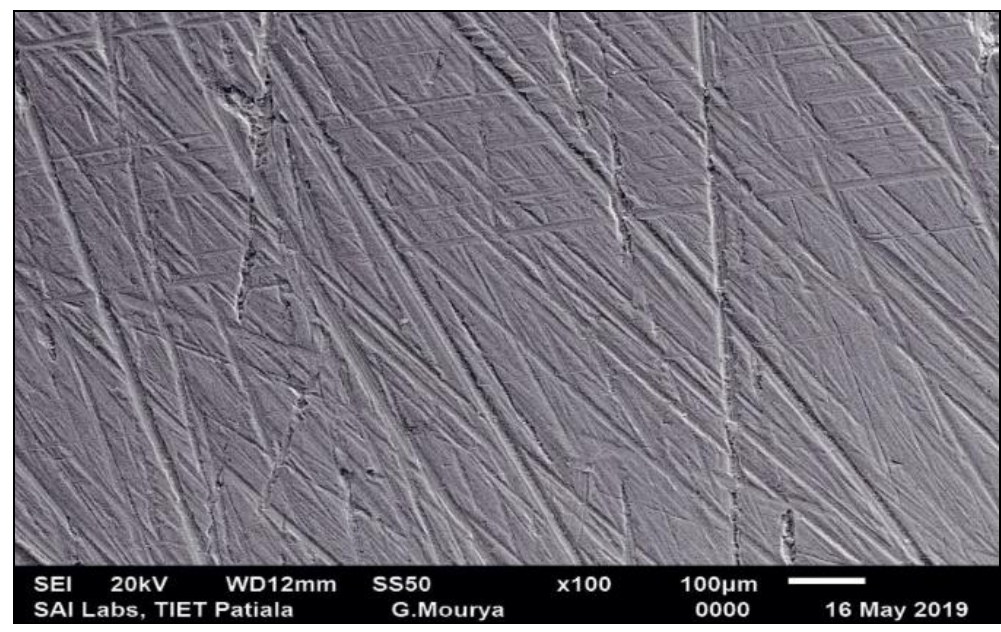

Fig. 8. SEM micrograph of workpiece before finishing at 100X, showing deep boring tool marks.

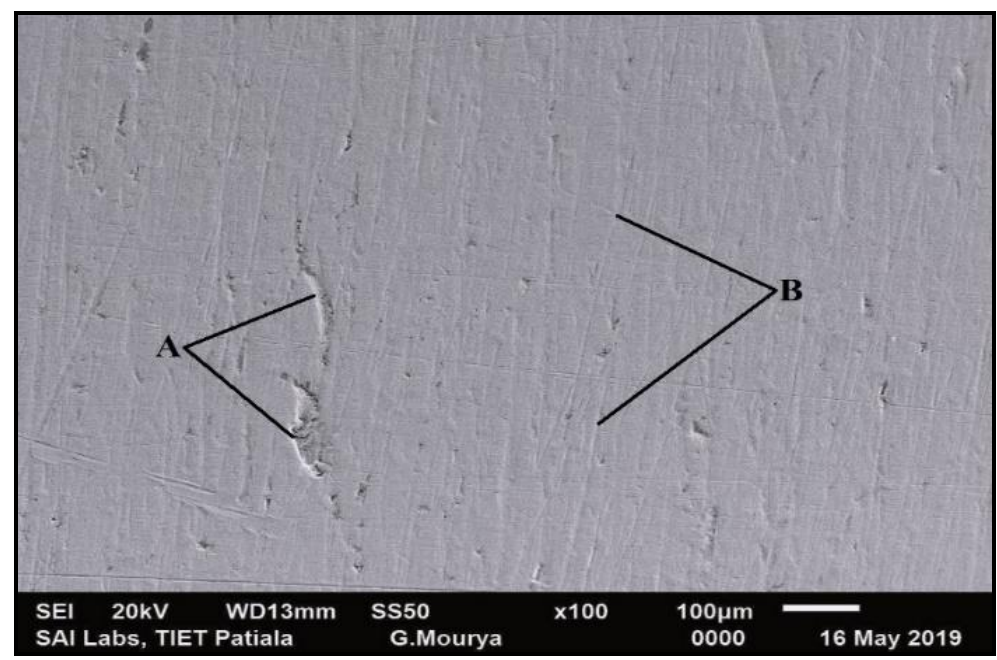

Fig. 9. SEM micrograph of workpiece finished with MAFF at 100X, A) shows some porosity in parent metal and B) shows abrasive grain marks.

\section{CONCLUSIONS}

A new method for producing bonded magnetic abrasive powder for finishing of aluminum tubes by magnetic abrasive flow finishing (MAFF) process has been proposed. Experiments were conducted in different finishing conditions to show the influence of variable parameters of finishing on final surface finish. It was concluded that:

1. The newly produced magnetic abrasive powder can be used as an alternative abrasive for achieving fine finishing on soft materials such as aluminum.

2. With increased magnetic flux density (MFD) from $0.15 \mathrm{~T}$ to $0.60 \mathrm{~T}$, surface finish improves, but after 0.6T of magnetic flux density (MFD), surface finish decreases. 
3. Improvement in surface finish (PISF) increased up to 5.6 $\mathrm{MPa}$ of extrusion pressure, but beyond 5.6 $\mathrm{MPa}$, the trend tends to change.

4. By increasing the number of working cycles from 10 to 25 , surface finish improved, but after this point by increasing working cycles to 30 , this result was inversed.

\section{ACKNOWLEDGMENT}

The research work reported here was made possible by the continuous support of BBSB Engineering College, Fatehgarh Sahib and department of RIC, IKGPTU Jalandhar.

\section{REFERENCES}

[1] Yamaguchi, H., Shinmura, T., Kobayashi, A.: JSME Int. J. Series C, vol. 44, no. 1, 2001, p. 275.

[2] Yin, S., Shinmura, T.: J. Japan Soc. Abrasive Technol., vol. 46, no. 3, 2002, p. 141.

[3] Bando, S., Tsukada, A., Kondo, Y.: J. Japan Soc. Abrasive Technol., vol. 45, no. 1, 2001, p. 46.

[4] Mehrdad, V., Nader, V.: J. Vac. Sci. \& Technol., vol. 27, no. 3, 2009, p. 1503.

[5] Chang, GW., Yan, BH., Hsu, RT.: Int. J. Mach. Tools \& Manuf., vol. 42, 2002, p. 575.

[6] Singh, L., Khangura, SS., Mishra, PS.: Int. J. Abrasive Technol., vol. 3, no. 3, 2010, p. 215.

[7] Singh, P., Singh, L., Singh S.: Powder Metallurgy Progress, vol. 19, no. 2, 2019, p. 82.

[8] Yamaguchi, H., Hanada, K.: J. Manuf. Sci. \& Eng., vol. 130, 2008, p. 031107.

[9] Jain, VK., Adsul, SG.: Int. J. Mach. Tools \& Manuf., vol. 40, 2000, p. 1003.

[10] Jha, S., Jain, VK.: Int. J. Mach. Tools \& Manuf., vol. 44, 2004, p. 1019.

[11] Kar, KK., Ravikumar, NL., Kumar, P., Tailor, B., Ramkumar, J., Sathiyamoorthy, D.: J. Mater. Processing Technol., vol. 209, 2009, p. 2212.

[12] Kenda, J., Pusavec, F., Kermouche, G., Kopac, J.: CIRP Conference on Surface Integrity (CSI), vol. 19, 2011, p. 172.

[13] Singh, S., Shan, HS.: Int. J. Mach. Tools \& Manuf., vol. 42, 2002, p. 953.

[14] Singh, S., Shan, HS., Kumar, P.: J. Mater. Processing Technol., vol. 128, 2002, p. 155.

[15] Sankar, MR., Ramkumar, J., Jain, VK.: Wear 266, 2009, p.688.

[16] Tzeng, HJ., Yan, BH., Hsu, RT., Chow, HM.: Int. J. Adv. Manuf. Technol., vol. 34, 2007, p. 649.

[17] Sran, LS., Khangura, SS., Singh, A.: In ASME International Manufacturing Science and Engineering Conference Notre Dame Indiana USA, 2012, p. 933.

[18] Patil, MG., Chandra, K., Mishra, PS.: Int. J. of Sci. Eng. \& Res., vol. 3, no. 10, 2012, p. 1.

[19] Singh, A., Singh, S., Singh, L.: Int. J. Adv. Manag. Technol. Eng. Sci., vol. 7, no. 11, 2017, p. 246.

[20] Dehghan, GA., Vahdati, M.: J. Eng. Manuf.: Part B, vol. 229, no. 9, 2015, p. 1517.

[21] Sankar, MR., Jain, VK., Ramkumara, J., Joshi, YM.: Int. J. Mach. Tools \& Manuf., vol. 51, no. 12, 2001, p. 947.

[22] Sachin, S., Deepu, K., Ravi, SM., Jain, VK.: Int. J. Adv. Manuf. Technol., vol. 100, no. 5-8, 2018, p. 1165. 